INTEGRATED ISLET DISTRIBUTION PROGRAM

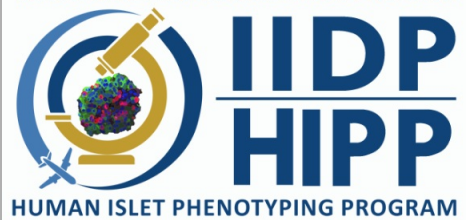

DEC 18, 2020

open ठaccess

DOI:

dx.doi.org/10.17504/protocol s.io.bppwmmpe

Protocol Citation: Human Islet Phenotyping Program (HIPP) of the IIDP 2020. Qualitative Assessment of Islet Viability by Staining with Fluorescein Diacetate (FDA) and Propidium lodide (PI) Dyes. protocols.io https://dx.doi.org/10.17504/p rotocols.io.bppwmmpe

License: This is an open access protocol distributed under the terms of the Creative Commons Attribution License, which permits unrestricted use, distribution, and reproduction in any medium, provided the original author and source are credited

Protocol status: Working We use this protocol and it's working

Created: Nov 13, 2020

Last Modified: Dec 18, 2020

PROTOCOL integer ID: 44502

\section{(3) Qualitative Assessment of Islet Viability by Staining with Fluorescein Diacetate (FDA) and Propidium lodide (PI) Dyes}

\author{
Human Islet Phenotyping Program (HIPP) of the \\ IIDP 1

\section{${ }^{1}$ Integrated Islet Distribution Program and Human Islet Phenotyping Program}

IIDP-HIPP

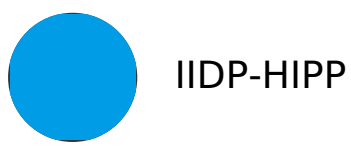

\section{ABSTRACT}

This Standard Operating Procedure (SOP) is adapted from the Viability SOP from the Clinical Islet Transplant Consortium Protocol and the Integrated Islet Distribution Program (IIDP) for Distribution Centers. This SOP provides the Human Islet Phenotyping Program (HIPP) procedures for Fluorescein Diacetate (FDA) and Propidium lodide (PI) staining and imaging of islet preparation for qualitative viability assessment.

This SOP defines the assay method used by the Human Islet Phenotyping Program (HIPP) for quantitative and qualitative determination of the Purified Human Pancreatic Islet product, post-shipment, manufactured for use in the National Institute of Diabetes and Digestive and Kidney Diseases (NIDDK)-sponsored research in the Integrated Islet Distribution Program (IIDP).

Note

This Standard Operating Procedure (SOP) \#: HIPP-03-v02

\section{GUIDELINES}

1. Integrated Islet Distribution Program (IIDP) (RRID:SCR 014387): The IIDP is a grant funded program commissioned and funded by the NIDDK to provide quality human islets to the diabetes research community to advance scientific discoveries 
Keywords: HIPP, fluorescein diacetate (FDA), propidium iodide $(\mathrm{PI})$, diabete and translational medicine. The IIDP consists of the NIDDK Project Scientist and Program Official, the External Scientific Panel and the $\mathrm{CC}$ at City of Hope $(\mathrm{COH})$. The IIDP CC integrates an interactive group of academic laboratories including the subcontracted IIDP centers.

2. IIDP Coordinating Center (CC): Joyce Niland, Ph.D. and Carmella Evans-Molina, M.D., Ph.D. serve as Co-Principal Investigators (Co-PIs) for the IIDP Program located within the Department of Diabetes and Cancer Discovery Science at $\mathrm{COH}$ to coordinate the activities of the IIDP and Human Islet Phenotyping Program (HIPP). Dr. Niland, contact PI, oversees the daily activity of the IIDP staff, provides informatics/ biostatistical input, and subcontracts with the Islet Isolation Centers (IICs) to ensure the delivery of the highest quality human islets to IIDP-approved investigators.Dr. Evans-Molina serves as the liaison to the HIPP, interacting closely to ensure that extensive, high quality phenotypic data are collected on islets distributed by the IICs. She also facilitates the delivery of this information to both the IICs and the IIDP-approved investigators, while responding to questions, issues, or suggestions for further HIPP enhancements.

3. Human Islet Phenotyping Program (HIPP): The HIPP is a subcontracted entity of the IIDP through the $\mathrm{COH}$ and Vanderbilt University. The HIPP is directed by Marcela Brissova, Ph.D. and is responsible for performing specific standardized quality control assays agreed upon by both the IIDP and the HIPP, in order to provide enhanced, quality data on the human islets post-shipment, to the IIDP. The results of these assays will be approved by the CC and posted on the IIDP website for both the centers and the approved investigators.

\section{Actual Islets (AI): The actual number of islets counted.}

5. Islet Equivalent (IEQ): An islet is quantified as $150 \mu \mathrm{m}$ diameter by mathematically compensating for the volume of the islet.

6. Fluorescein Diacetate/Propidium lodide (FDA)/(PI) Viability Assay is a rapid fluorometric method to test the integrity of the plasma membrane simultaneously using inclusion and exclusion dyes; the assay differentiates between viable and nonviable cells and is, consequently, used for determination of viability of islet preparations.

6.1 The inclusion dye is fluorescein diacetate (FDA) and the exclusion dye is propidium iodide $(\mathrm{PI})$. The final concentrations are as follows:

$$
\begin{gathered}
\text { FDA: } 0.46 \mu \mathrm{M} \\
\text { PI: } 14.34 \mu \mathrm{M}
\end{gathered}
$$

6.2 Fluorescein diacetate is a nonpolar ester, which passes through plasma membranes and is hydrolyzed by intracellular esterases to produce free fluorescein. 
The polar fluorescein is confined within cells with an intact plasma membrane and can be observed under appropriate excitation conditions. FDA functions as an inclusion dye, i.e., viable cells will appear bright green fluorescent using FDA.

6.3 Propidium iodide functions as an exclusion dye that cannot penetrate living cells but readily enters dead or dying cells. Once PI penetrates through the cell membrane, it binds to nucleic acids and causes them to fluoresce bright orange/red. $\mathrm{PI}$ absorbs in green light and fluoresces orange/red.

\section{References:}

\section{CITATION}

Bank, HL (1987). Assessment of Islet Cell Viability Using Fluorescent Dyes. Diabetologia, 30:812-816.

LINK

https://pubmed.ncbi.nlm.nih.gov/3322907/

\section{CITATION}

Bank, HL (1987). Rapid Assessment of Islet Viability with Acridine Orange and Propidium Iodide. Invitro Cellular \& Developmental Biology, 24:4, pp. 266-273.

LINK

https://pubmed.ncbi.nlm.nih.gov/3284875/

\section{CITATION}

Ricordi, C. Pancreatic Islet Cell Transplantation. Austin: R.G. Landes Company, 1992:137138.

\section{MATERIALS}

\section{Equipment:}


1. Fluorescent Microscope

2. Calculator or computer software (e.g. Excel) with the mean and standard deviation functions

\section{Equipment}

Fluorescence Microscope

Olympus BX50 DIC

Olympus

BX50-DIC-FL

https://www.olympus-lifescience.com/en/microscoperesource/primer/techniques/fluorescence/fluorhome/

\section{Supplies and Materials:}

1. Fluorescein diacetate, stock solution $24 \mu \mathrm{M}(9.9 \mu \mathrm{g} / \mathrm{mL}$ in acetone $)$, Sigma, Cat. \#F-7378, or equivalent

2. Propidium iodide, stock solution $750 \mu \mathrm{M}(0.5 \mathrm{mg} / \mathrm{mL}$ in DPBS, $\mathrm{pH}$ approximately 7.4), Sigma, Cat. \#P-4170, or equivalent

3. Acetone, Sigma, Cat. \#179124, or equivalent

4. Sterile $10 \times 35 \mathrm{~mm}$ cell culture dishes, Nunc Cat. \#174926, or equivalent

5. DPBS (Dulbecco's Phosphate Buffered Saline) without calcium or magnesium, Mediatech, Part \#21-031, or equivalent

6. Pipettes: 200 and $1000 \mu \mathrm{L}$ with associated tips (P-200 ART and P-1250 ART) 
88 Fluorescein diacetate Merck MilliporeSigma (Sigma-Aldrich) Catalog \#F-7378, equivalent

88

Propidium iodide Merck MilliporeSigma (Sigma-Aldrich) Catalog

\#P4170

88

Acetone Merck MilliporeSigma (Sigma-Aldrich) Catalog

\#179124

88

Dulbecco's Phosphate-Buffered Saline (without calcium and

magnesium) Corning Catalog \#21-031

\section{Equipment}

Pipettes

NAME

200 and 1000 uL with associated tips

TYPE

Fisher Scientific

BRAND

None

SKU

https://www.fishersci.com/us/en/products/19C8L3D9/pipets-pipetters-

LINK tips.html

\section{Equipment}

pipette tips

NAME

P-200 ART and P-1250 ART

TYPE

ART

BRAND

A3098

SKU

https://www.sigmaaldrich.com/catalog/product/sigma/a3098?

lang=en\&region=US 
Note

Sigma-Aldrich, (RRID:SCR_008988)

Thermo Fisher Scientific, (RRID:SCR_008452) 
Always wear gloves and observe standard chemical procedures:

Fluorescein Diacetate: [C FDA MSDSAction.pdf

Safety information

Protect from light. Avoid contact and inhalation. Nitrile gloves are recommended in the MSDS when handling FDA.

Propidium lodide: [f PI MSDSAction.pdf

\section{Safety information}

Use personal protective equipment. Product may be toxic if inhaled, swallowed, or splashed on skin. Avoid dust formation. Avoid breathing vapors, mist, or gas. Ensure adequate ventilation. Wear gloves and observe Safety Data Sheet. Suspected of causing genetic defects.

\section{Acetone: @ AcetoneMSDSAction.pdf}

\section{Safety information}

Solvent/Flammable. Keep away from heat, spark, and open flame. Keep container tightly closed. Use with adequate ventilation. Avoid contact with eyes. Avoid prolonged or repeated breathing of vapor. Handle with gloves. Gloves must be inspected prior to use. Use proper glove removal technique (without touching glove's outer surface) to avoid skin contact with this product. Dispose of contaminated gloves after use in accordance with applicable laws and good laboratory practices. Wash and dry hands. Store aliquots in tightly sealed in glass or polypropylene tubes with polypropylene or polyethylene closure. 


\section{Procedures}

Limitations

1.1 Once the dye is added to the islets, the assessment must take place as quickly as possible. If there is a delay of more than 15 minutes, the accuracy of the assessment will be diminished as the islets lose their viability with time.

1.2 Both of the fluorescent dyes used in this assay are light sensitive and must be kept in the dark, covered with aluminum foil.

1.3 The fluorescent dyes are temperature sensitive and must be stored as follows:

- FDA: $\leq-20^{\circ} \mathrm{C}$

- PI: $2-8^{\circ} \mathrm{C}$

$2 \quad$ Assay Set Up

2.1 Assemble all items described in the "Supplies and Materials" section.

2.2 Prepare Fluorescent dyes: Fluorescein Diacetate (FDA) and Propidium lodide (PI), if required.

\section{Preparation of Fluorescein Diacetate and Propidium lodide}


3.1 Remove FDA from the freezer and PI from the refrigerator. Weigh the required amount of the reagent on an analytical balance.

3.2 Dissolve $\triangle 0.00199 \mathrm{~g}$ of FDA in $\triangle 200 \mathrm{~mL}$ of acetone in a glass bottle and cover with aluminum foil. Store in $\triangle 10 \mathrm{~mL}$ aliquots at $-20^{\circ} \mathrm{C}$.

3.3 Dissolve $\triangle 0.0125 \mathrm{~g}$ of PI in $\triangle 25 \mathrm{~mL}$ of DPBS and cover with aluminum foil. Store in I $5 \mathrm{~mL}$ aliquots at $2-8^{\circ} \mathrm{C}$.

3.4 Discard used stain.

3.5 Record the expiration date on each aliquot tube. The expiration date, for both PI and FDA stains, is six months from the date of preparation.

\section{$4 \quad$ Staining and Estimation of Viability}

4.1 Add two $\triangle 400 \mu \mathrm{L}$ replicates of well-mixed total islet suspension to the culture dish.

4.2 Quickly add first $\triangle 10 \mu \mathrm{L}$ of PI and then $\triangle 10 \mu \mathrm{L}$ of FDA to the islet suspension. Gently swirl to mix.

4.3 Turn off the lights in the room. 
4.4 Incubate plate in the dark for 15 minutes.

4.5 Assess the preparation immediately using the fluorescent microscope. Image islets using an Olympus SZX12 stereomicroscope system. Capture multiple fields ensuring that FDA/PI staining is visualized in $50-100$ islets per preparation.

Note

FDA produces bright green fluorescence in viable cells, while PI produces red fluorescence in dead or dying cells.

\section{$5 \quad$ Interpretation of Results}

5.1 FDA freely passes through the cell membrane of live cells. Viable cells appear bright fluorescent green when stained with FDA. In a live cell, FDA is hydrolyzed to the polar free fluorescein, and it is trapped within the intact membranes of the viable cells present in islets.

5.2 PI stains the nuclei of dead/non-viable cells only. Dead cells appear bright fluorescent red/orange. PI does not cross the membrane of viable cells.

5.3 FDA/PI staining captures viable and dead cells in both islets and residual exocrine tissue. This staining provides a rapid qualitative viability assessment of the human islet preparation; however, the 3-D nature of islets and exocrine tissue remnants precludes accurate cell counting. The Vanderbilt HIPP will supplement viability assessment with counts of live and dead cells in a single cell suspension using Countess II technology (Life Technologies AMQAX1000) (see HIPP Protocol-04-v02 Quantitative Assessment of Islet Viability by Trypan Blue Staining). 


\section{Data Storage and Reporting}

6 To facilitate data management and ensure data security, the Vanderbilt HIPP uses an institutional server-based platform for data storage and analysis.

7 Annotated images containing metadata (Figure 1) will be uploaded to the IIDP-HIPP database and immediately disseminated to IIDP-affiliated investigators and islet isolation centers.
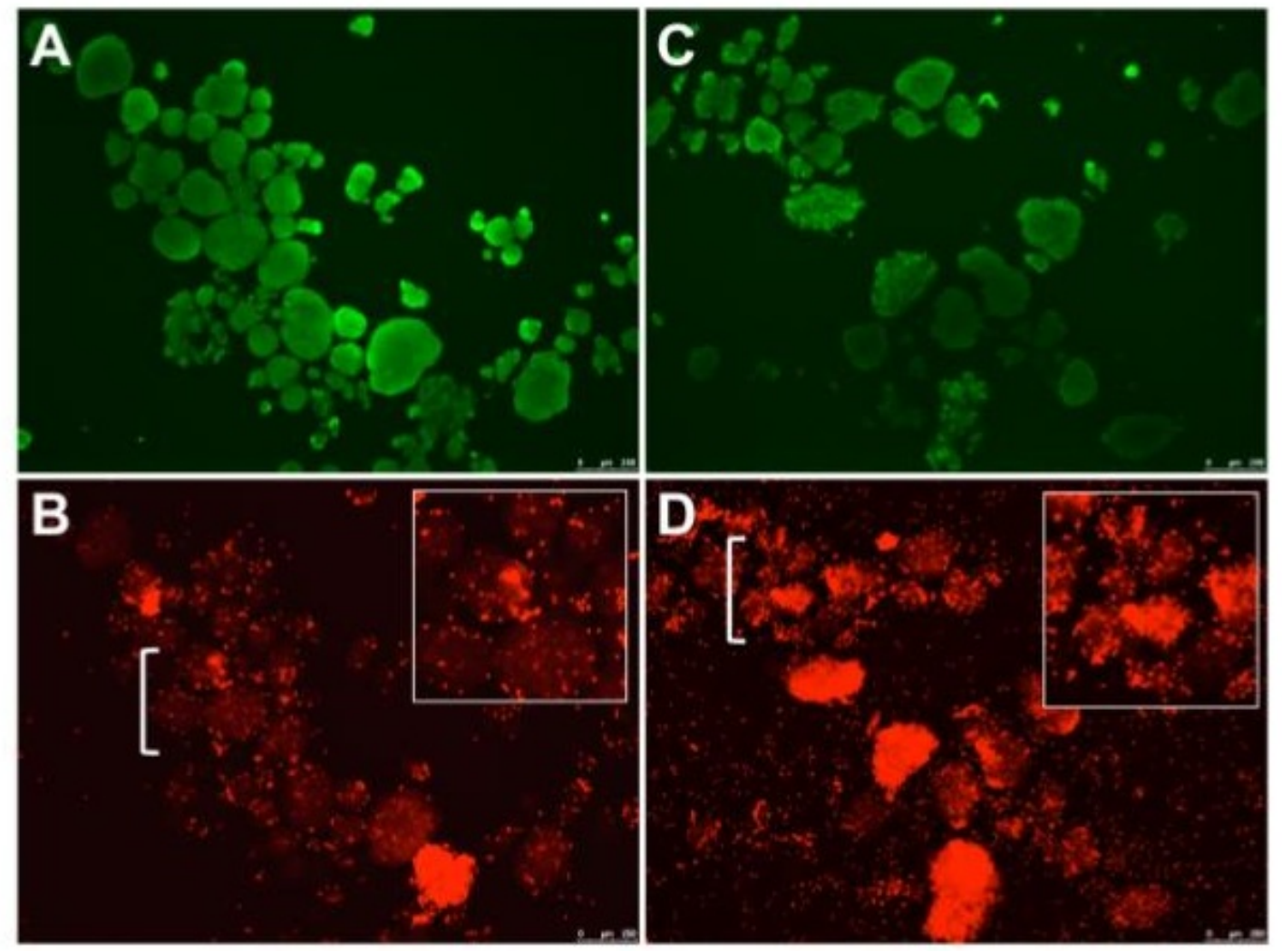

Figure 1. Qualitative viability assessment of human islet preparations by FDA/PI staining. Two human islet preparations; preparation \#1 (A, B) and preparation \#2 (C, D) were stained side-byside with FDA and PI dyes. FDA staining (green) appeared to be weaker in preparation \#2 compared to preparation \#1. Preparation \#1 had less PI labeling (red) in cell clusters and fewer $\mathrm{PI}+$ single cells compared to preparation \#2. Brackets in panels $\mathbf{B}$ and $\mathbf{C}$ indicate the region magnified in respective insets.

\section{Deviations and Resolutions:}


8 Document any deviations that occurred from this protocol that affect the final results and report with the analysis of the assay. 